\title{
Effect of gut microbiome on minor complications after a colonoscopy
}

\author{
Jae Hyun Kim, Youn Jung Choi, Hye Jung Kwon, Kyoungwon Jung, Sung Eun Kim, Won Moon, Moo In Park, \\ Seun Ja Park \\ Department of Internal Medicine, Kosin University College of Medicine, Busan, Korea
}

Background/Aims: Minor complications that might occur after colonoscopy, including abdominal discomfort, bloating, diarrhea, and constipation, could a barrier for patients to undergo a screening colonoscopy. In this study, we aimed to identify the effect of gut microbial diversity and composition on minor complications after colonoscopy. Methods: A total of 24 healthy subjects provided their stools before bowel preparation and on the 7th and 28th day after colonoscopy. On the 7th day after colonoscopy, the presence of minor complications was investigated using a questionnaire. We divided patients into 2 groups, the no complication group and complications group. The fecal microbial diversity, distribution, and composition were then compared between the groups. Results: Five of the 24 subjects reported that they had undergone minor complications after colonoscopy. Most of the symptoms were mild and self-limited, but 1 patient needed medication. Interestingly, the Firmicutes/ Bacteroidetes ratio of the initial stool samples before bowel preparation in the complication group was significantly higher than that in no complication group. After bowel preparation, the Firmicutes/Bacteroidetes ratio of the complication group decreased, but not in the no complication group. The microbial diversity of the no complication group decreased after bowel preparation, but not in the complication group. Conclusions: The gut microbial composition and diversity before and after bowel preparation could be considered as one of the causes of minor complications after colonoscopy. Further studies are needed to delineate the role of gut microbiota in the occurrence of minor complications after colonoscopy. (Intest Res 2021;19:341-348)

Key Words: Gut microbiota; Adverse effects; Cathartics; Colonoscopy

\section{INTRODUCTION}

Colonoscopy is a useful method for detecting and removing colorectal polyps, thereby reducing the incidence of colorectal cancer. The U.S. Preventive Service Task Force recommends screening colonoscopy for colorectal cancer from the age of 50 years old. ${ }^{1}$ However, the adherence to screening colonoscopy is lower than another tool, a fecal occult blood test. In a randomized controlled trial, the overall participation rates for screening colonoscopy was only $38 \%$, compared with $67 \%$ for

Received May 22, 2020. Revised June 17, 2020. Accepted June 30, 2020. Correspondence to Seun Ja Park, Department of Internal Medicine, Kosin University College of Medicine, 262 Gamcheon-ro, Seo-gu, Busan 49267, Korea. Tel: +82-51-990-5061, Fax:+82-51-990-5055, E-mail: parksj6406@ daum.net a fecal occult blood test. ${ }^{2}$ Patient-reported barriers to screening colonoscopy included fear of pain and concerns about bowel preparation and complications. ${ }^{3}$ Colonoscopy is associated with potentially serious complications of perforation and hemorrhage, as well as minor complications, including abdominal discomfort, bloating, nausea, diarrhea, and constipation. ${ }^{4,5}$ The incidence of minor complications after colonoscopy has been reported as between $24 \%$ and $32 \%$, with most minor complications occurring within 7 days. ${ }^{5,6}$

In recent years, the interest in the gut microbiome has increased and has attracted attention as the cause of various symptoms. The colon has the largest and most diverse bacterial flora in the human body, which is involved in digesting food, synthesizing vitamins and amino acids, preventing pathogen formation, regulating the immune system, and secretion of 
gastrointestinal hormones and brain activity. ${ }^{7-9}$ In particular, dysbiosis of the gut microbiome have been demonstrated in various conditions including metabolic diseases, inflammatory diseases and cancers. ${ }^{10-12}$ Recently, several studies have shown that bowel preparation for colonoscopy could change the fecal microbial diversity and composition, and these effects could last up to 1 month. ${ }^{13-15}$ Bowel preparation also affects the change of gut metabolome but this is recovered within 14 days. ${ }^{16}$ To minimize these effects, bowel preparation using 2 separate dosages could be helpful as this results in fewer alterations to the gut microbiome than a single dose. ${ }^{17}$

In this study, we hypothesized that the gut microbial diversity and composition before and after bowel preparation could affect the occurrence of minor complications after a colonoscopy. We aimed to identify the effect of gut microbiome on minor complications that might occur after a colonoscopy.

\section{METHODS}

\section{Subjects and Stool Collection}

The study protocol was approved by the Kosin University Gospel Hospital (IRB No. KUGH 2017-07-036). Between May 2018 and July 2019, a total of 24 healthy subjects were enrolled in this study conducted at Kosin University Gospel Hospital, Busan, Korea. Patients with a history of abdominal surgery, inflammatory bowel disease, or cancer were excluded. Patients who had taken laxatives, metoclopramide, tegaserod, proton pump inhibitor, or antibiotics within the month prior to sampling were also excluded. An investigator (J.H.K.) explained the aim and contents of the study in detail to the participants. All participants provided written informed consent before enrollment in this study. Their information, such as age, sex, alcohol and smoking history, comorbidities, previous colonoscopy experience, the reason for a colonoscopy, symptoms before colonoscopy including abdominal discomfort, bowel habit changes, hematochezia or melena, constipation or diarrhea, were collected using a questionnaire. Before bowel preparation for colonoscopy, all participants collected a stool using a fecal sample collector kit (Medi4U, Incheon, Korea) at home and were informed to store it immediately after collection at $-20^{\circ} \mathrm{C}$ in a freezer. As soon as possible, the samples were transported to the laboratory of Kosin University Gospel Hospital, enclosed in an insulated foil pack with dry ice, and stored at $-80^{\circ} \mathrm{C}$ in a deep-freezer. On the 7th and 28th day after the colonoscopy, all participants collected and submitted their stool in the same way. Af- ter the collection of all samples, we transported them to Bioeleven Co., Ltd. (Seoul, Korea) to perform the analysis. On the 7th day after the colonoscopy, a questionnaire asking about any symptoms suffered was provided to participants. This included type, timing, frequency, and duration of the symptoms and was used to evaluate the occurrence of minor or severe complications after colonoscopy.

\section{Bowel Preparation and Colonoscopy}

All participants ingested $2 \mathrm{~L}$ of Coolprep (polyethylene glycol [PEG] solution with $20 \mathrm{~g}$ ascorbic acid; Taejoon Pharm, Seoul, Korea) between 7 and 9 PM on the day before the colonoscopy and the remaining $2 \mathrm{~L}$ in the morning at least 2 hours before the colonoscopy. Colonoscopic examinations were performed by 2 experienced endoscopists (Y.J.C. and H.J.K.). During the examination, the insertion time, withdrawal time, bowel preparation scale, and polyp detection were assessed. When a polyp was detected during the colonoscopic examination, a biopsy or polypectomy and histologic assessments were performed to identify the adenoma component.

\section{Sample Preparation and Data Analysis}

The total bacterial DNA was extracted from $200 \mathrm{mg}$ of the stool sample using a QIAamp Fast DNA Stool Mini Kit (Qiagen, Hilden, Germany), following the manufacturer's instructions. The DNA concentration was measured using an ultraviolet-vis spectrophotometer (NanoDrop 2000c; Thermo Fisher Scientific, Waltham, MA, USA) and quantified using a QuantiFluor ${ }^{\circledR}$ ONE dsDNA System (Promega, Madison, WI, USA). All extracted DNA samples were stored at $-20^{\circ} \mathrm{C}$ until use in further experiments. The V3-V4 region of the bacterial 16S rRNA gene was amplified by primer sets F319 (5'-TCGTCGGCAGCGTCAGATGTGTATAAGAGACAGCCTACGG-GNGGCWGCAG) and R806 (5'-GTCTCGTGGGCTCGGAGATGTGTATAAGAGACAGGACTACHVGGGTATC-TAATCC-3'). The polymerase chain reaction (PCR) mixture contained $12.5 \mathrm{ng} / \mu \mathrm{L}$ DNA template, the KAPA HiFi Hotstart PCR Kit (Kapa Biosystems, Woburn, MA, USA), and $5 \mu \mathrm{M}$ of each primer. PCR products were examined on a $2 \%$ agarose gel electrophoresis and purified using an AMPure XP magnetic bead (Beckman Coulter, High Wycombe, UK) based purification. The quality of purified products was measured by the Agilent Bioanalyzer (Agilent, Santa Clara, CA, USA). A secondary amplification to attach the Illumina Nextera barcodes (Illumina, Inc., San Diego, CA, USA) was then carried out using the i5 forward primer and the i7 reverse primer. The amplified products were puri- 
fied using AMPure XP magnetic beads (Beckman Coulter) according to the manufacturer's protocol. The purified products were quantified using a QuantiFluor ${ }^{\circledR}$ ONE dsDNA System (Promega). The product size and quality were evaluated on a Bioanalyzer 2100 (Agilent). The pooled libraries were sequenced using an Illumina MiSeq instrument with a MiSeq v3 Reagent Kit (Illumina, Inc).

\section{Statistical Analysis}

The data of the 16S rRNA gene sequences were quality filtered, aligned to the Silva database, and screened using the software mothur. ${ }^{18,19}$ Chimeric sequences were removed using uchime, ${ }^{20}$ and artificial erroneous reads were corrected using the pre cluster mothur subroutine. Taxonomic classification was carried out using the Ribosomal Database Project (training set version 14). ${ }^{21}$ The non-archaeal/bacterial sequence was removed based on the taxonomic classification results. Operational taxonomic units (OTUs) were estimated at a distance of 0.03 using the mothur subroutine cluster split. Alpha and beta-diversity analyses were used to assess biodiversity based on OTUs and visualized by ggplot utilizing the $\mathrm{R}$ software package ( $\mathrm{R}$ Foundation for Statistical Computing, Vienna, Austria).

\section{RESULTS}

\section{Baseline Characteristics and Colonoscopy Outcomes}

A total of 24 subjects was enrolled in this study. Their baseline characteristics and colonoscopy outcomes are summarized in Table 1. The mean age of all participants was $42.8 \pm 11.9$ years old, and 10 subjects (41.7\%) were male. Eight subjects reported their reason for colonoscopy as abdominal discomfort, constipation, loose form stools, or anal bleeding, whereas 16 subjects wanted to undergo a screening colonoscopy. During the colonoscopic examination, the mean insertion time was $267 \pm 138$ seconds, the mean withdrawal time was $687 \pm 320$ seconds, and the cecal intubation success rate was $100 \%$. Colon polyps were detected in 11 subjects (average number of polyps per subject; $2.7 \pm 1.7$ ). All detected polyps were removed by polypectomy.

\section{Minor Complications after Colonoscopy}

During the colonoscopic examination, immediate complications, including bleeding and perforation, did not occur. Delayed bleeding and perforation were also not reported. In the questionnaire on the 7th day after the colonoscopy, 5 of the 24 subjects (20.8\%) reported that they had undergone minor complications, including abdominal pain, abdominal disten-
Table 1. Baseline Characteristics and Colonoscopic Outcomes of Enrolled Subjects

\begin{tabular}{lc}
\hline Variable & Total $(\mathrm{n}=24)$ \\
\hline Mean age (yr) & $42.8 \pm 11.9$ \\
Male sex & $10(41.7)$ \\
\hline Alcohol history & $9(37.5)$ \\
Current smoker & $3(12.5)$ \\
Diabetes mellitus & 0 \\
Hypertension & $3(12.5)$ \\
Dyslipidemia & $1(4.2)$ \\
Reason for colonoscopy & \\
Screening & $16(66.7)$ \\
Abdominal discomfort & $4(16.8)$ \\
Constipation & $1(4.1)$ \\
Loose form stools & $1(4.1)$ \\
Anal bleeding & $2(8.3)$ \\
Colon polyps & $11(45.8)$ \\
No. of polyps per subject & $2.7 \pm 1.7$ \\
Cecal intubation success & $24(100)$ \\
Insertion time (sec) & $267 \pm 138$ \\
Withdrawal time (sec) & $687 \pm 320$ \\
Total examination time (sec) & $954 \pm 458$ \\
\hline
\end{tabular}

Values are presented as the mean \pm standard deviation or number (\%).

sion, constipation or diarrhea. Only 2 of the 8 subjects who had any symptoms at the time of enrollment had minor complications after colonoscopy. Most of the symptoms did not last up to 24 hours, but 1 patient needed medication including probiotics, to relieve their symptoms. The reported minor complications of the 5 subjects are summarized in Table 2 .

\section{Correlation between Minor Complications and Microbial Diversity and Distribution}

We hypothesized that gut microbiota could affect the occurrence of minor complications after colonoscopy. All subjects were divided into 2 groups according to the absence or presence of minor complications; the no complication group and complication group, respectively. Then, we assessed the alphadiversity and beta-diversity to examine the microbial diversity and distribution of each stool sample. Stool samples were taken before bowel preparation, on the 7th day after colonoscopy and the 28th day after colonoscopy. As shown in Fig. 1, the alpha-diversity of the initial stool samples before bowel preparation in the no complication group was higher than that of the complication group. The microbial diversity of the no compli- 


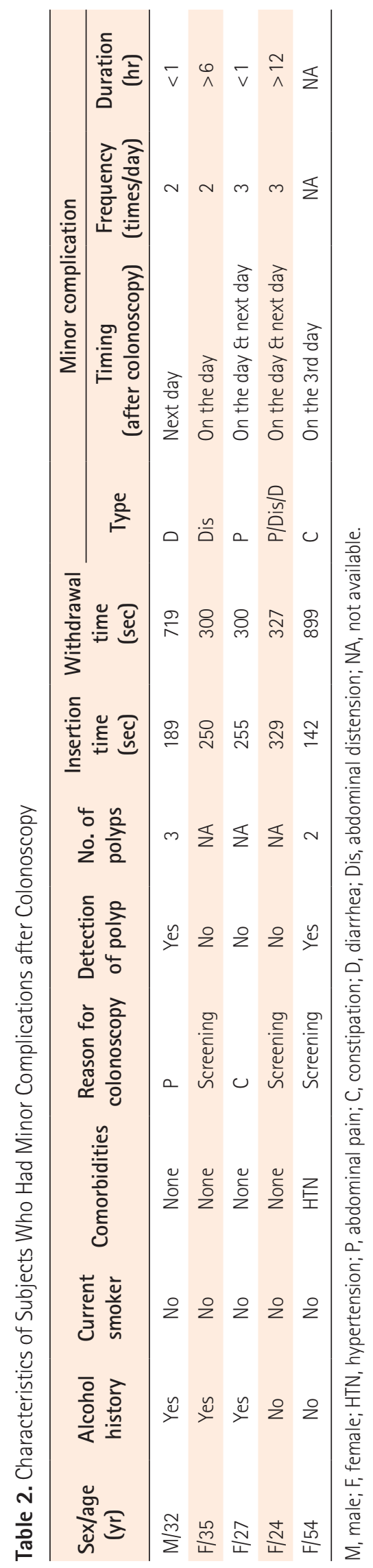

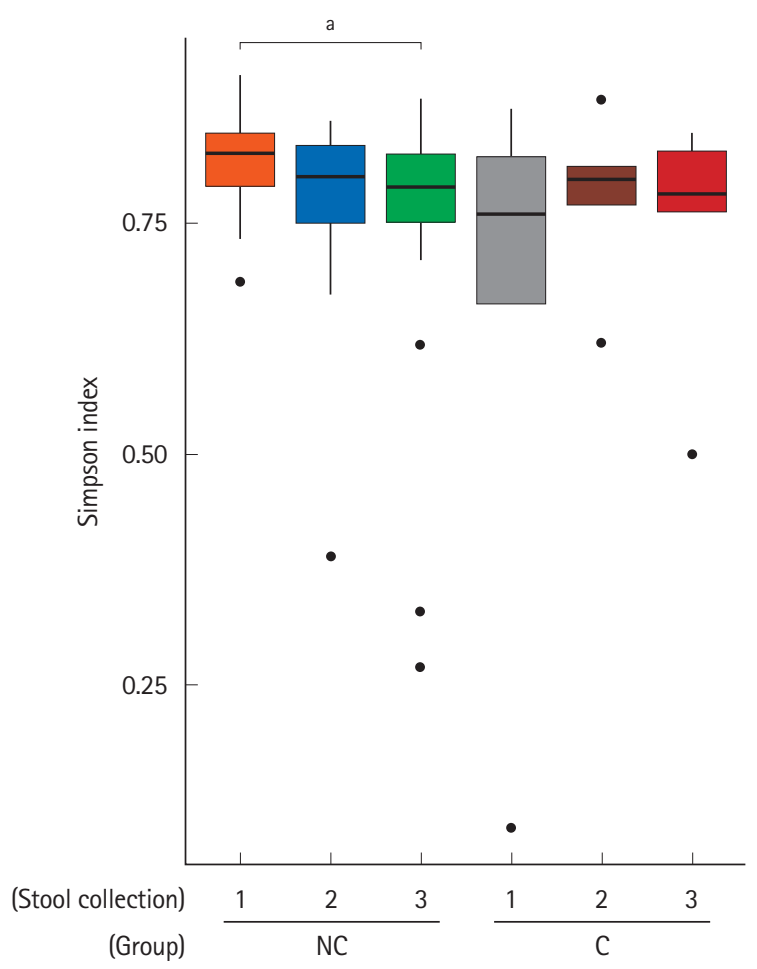

Fig. 1. Box plot for the alpha-diversity of bacterial communities in the 2 groups-no complication group (NC) and complication group (C)-according to the timing of the stool collection $(1$, before bowel preparation; 2 , on the 7 th day after colonoscopy; 3 , on the 28th day after colonoscopy). The box plot presents the full range of values obtained from the source data. The ggplot of the $\mathrm{R}$ software package was used for visualization $\left({ }^{a} P<0.05\right)$.

cation group gradually decreased after bowel preparation, whereas the microbial diversity of the complication group did not decrease after bowel preparation. In the analysis of microbial distribution using beta-diversity, each sample was relatively separated and showed a different distribution according to their group (Fig. 2).

\section{Correlation between Minor Complications and Microbial Composition}

Interestingly, in the comparison of microbial composition at the phylum level, we found that the Firmicutes/Bacteroidetes ratio of the initial stool samples before bowel preparation in the complication group was significantly higher than that in the no complication group (Figs 3, 4). As shown in Fig. 3, Firmicutes occupied most of the composition of the stool samples before bowel preparation in the complication group, and after bowel preparation, the Firmicutes/Bacteroidetes ratio decreased. The original Firmicutes/Bacteroidetes ratio had recovered by the 28th day after colonoscopy. Contrastingly, the Firmicutes/ 


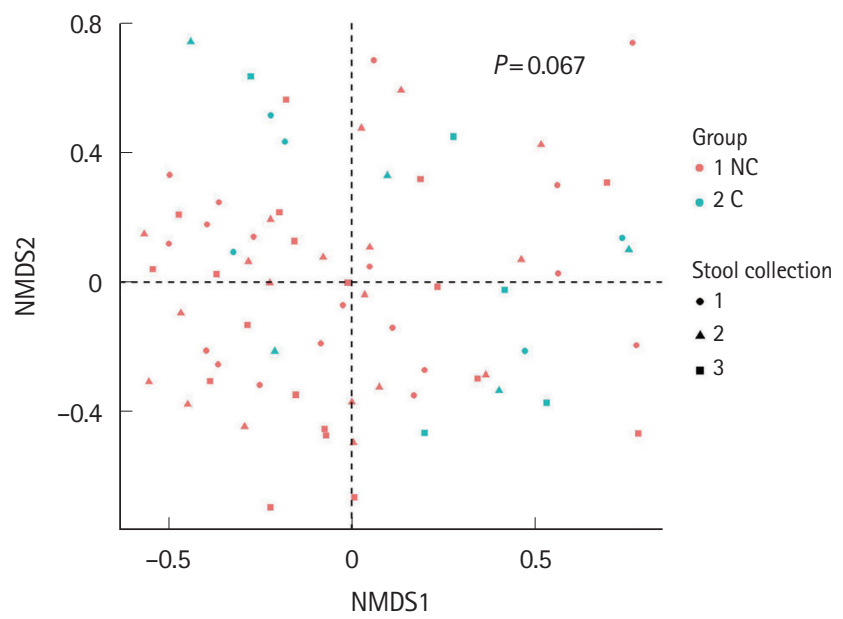

Fig. 2. Beta-diversity visualized using the non-metric multidimensional scaling (NMDS) plot with Bray-Curtis dissimilarity distances of the 2 groups-no complication group (NC) and complication group (C)-according to the timing of the stool collection $(1$, before bowel preparation; 2 , on the 7 th day after colonoscopy; 3 , on the 28th day after colonoscopy).

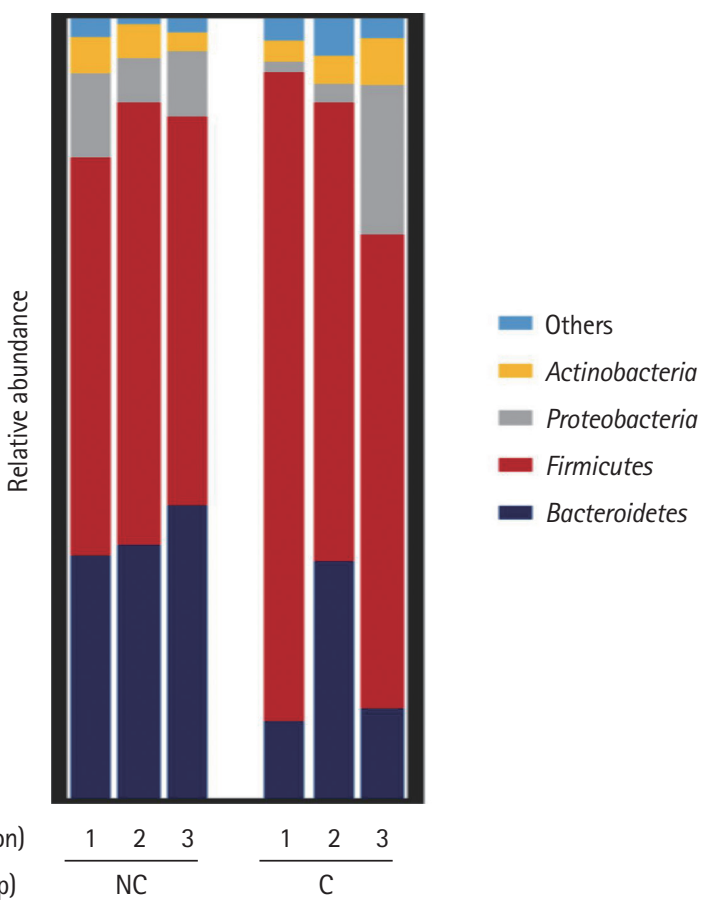

Fig. 3. Composition of bacterial communities at the phylum level in the 2 groups-no complication group (NC) and complication group (C)-according to the timing of the stool collection $(1$, before bowel preparation; 2 , on the 7th day after colonoscopy; 3 , on the 28th day after colonoscopy).

Bacteroidetes ratio in the no complication group did not change between the samples taken before bowel preparation and on

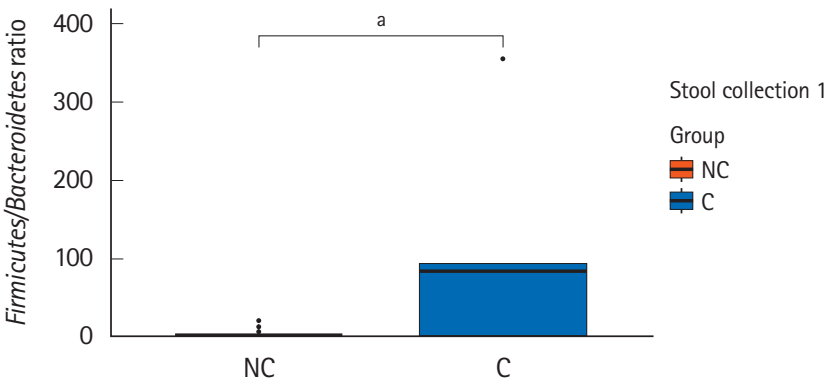

Fig. 4. Comparison of the Firmicutes/Bacteroidetes ratio of the initial stool sample before bowel preparation in the 2 groups -no complication group (NC) and complication group (C). ${ }^{2}$ Wilcoxon signed-rank test, $P=0.01$.

the 7th and 28th days after the colonoscopy. In the comparison of microbial composition at the genus level, the results show a similar pattern as those at the phylum level (Supplementary Fig. 1). The microbial composition at the phylum and genus level of all subjects are provided in Supplementary Fig. 2.

\section{DISCUSSION}

In this study, we found that the initial gut microbial composition and diversity before bowel preparation between groups that had or did not have minor complications after colonoscopy was different. The Firmicutes/Bacteroidetes ratio in the group who had minor complications decreased after bowel preparation. Based on these results, we propose that the distinct change of Firmicutes/Bacteroidetes ratio after bowel preparation in subjects who have initially possessed Firmicutes dominantly could affect the occurrence of minor complications after colonoscopy. Before bowel preparation, these subjects might need to correct their gut microbial composition that consists dominantly of Firmicutes. This could help to reduce minor complications after colonoscopy.

Bowel preparation for colonoscopy is commonly considered safe for a generally healthy person. Bowel preparation using solutions, such as PEG, causes an increase in the amount of water in the gastrointestinal tract and washes out the luminal contents, including fecal material. These alterations result in the bowel movements increasing to flush out bacteria and disrupt bacterial adherence to the gut mucosa. Bowel preparation could affect the quality and production of the protective mucus layer in the colon and cause diarrhea or constipation. ${ }^{22}$ After a colonoscopy, minor complications including abdominal discomfort, bloating, nausea, diarrhea, and constipation can occur. While the majority of these symptoms are mild and 
self-limited, patients who have adverse events after colonoscopy are less willing to undergo the procedure again., ${ }^{5,23}$ There are several explanations for the development of minor complications after colonoscopy. Increased sensitivity to colonic manipulation or poor bowel preparation results in a longer insertion time, which requires more air insufflation or additional technical manipulation to reach the cecum. Individuals found to have polyps or other abnormalities have longer biopsies or polypectomy. In this study, the total examination time including insertion time and withdrawal time in subjects with minor complications after colonoscopy was not longer than the mean total examination time of subjects except for in 1 individual. In subjects with minor complications after colonoscopy, 2 individuals underwent a polypectomy, but 3 individuals did not. All subjects with minor complications after colonoscopy had different baseline characteristics including age, sex, alcohol and smoking history, and comorbidities, as shown in Table 2.

The Firmicutes are a phylum of bacteria, which mostly have a Gram-positive cell wall structure. Firmicutes, along with Bacteroidetes, are one of the most abundant bacteria in the human gut. In several studies, the Firmicutes/Bacteroidetes ratio is seen to be increased in obese individuals compared to lean controls, and a higher abundance of Firmicutes has been linked to more adiposity and body weight. ${ }^{24-26}$ In patients with irritable bowel syndrome, the Firmicutes/Bacteroidetes ratio has been reported to be linked to both a higher and lower ratio. ${ }^{27,28}$ The ratio of Firmicutes/Bacteroidetes evolves throughout life and the alteration of the Firmicutes/Bacteroidetes ratio may be involved in metabolic diseases including obesity. ${ }^{29}$ In this study, we found that subjects with minor complications after colonoscopy had an initially high abundance of Firmicutes and the ratio of Firmicutes/Bacteroidetes decreased after bowel preparation. Although further studies are needed to evaluate the correlation with other factors related to colonoscopic examination, these findings suggest that the initial abundance of Firmicutes and distinct decrease of the Firmicutes/Bacteroidetes ratio after bowel preparation could affect the occurrence of minor complications after colonoscopy.

This study has several limitations. First, this study was conducted only on residents in a single center. Therefore, these results may not represent populations in other cities and countries. However, these data might contribute to the basic concept of correlations between gut microbiota and minor complications after colonoscopy. Second, 8 subjects enrolled in this study reported that they had symptoms such as abdominal discomfort, constipation, loose form stools, or anal bleed- ing before colonoscopy. However, their symptoms were not matched with Rome IV criteria for diagnosing irritable bowel syndrome. Third, we have not performed a regression analysis for the occurrence of minor complications, which assesses the association of gut microbiota and colonoscopy related outcomes, including colonoscope insertion and withdrawal time and colon polypectomy. Further studies are needed to clarify the relationship of these factors on minor complications after colonoscopy.

In conclusion, we found that gut microbial composition and diversity before and after bowel preparation could affect the occurrence of minor complications after colonoscopy. Based on our results, we propose that an assessment of gut microbiota before bowel preparation is needed. An intervention with probiotic treatment might be considered in subjects who possess Firmicutes dominant stools before bowel preparation. Further metagenome-wide association studies will be required to clarify the usefulness of evaluating gut microbiota on the occurrence of minor complications after colonoscopy.

\section{ADDITIONAL INFORMATION}

\section{Funding Source}

This work was supported by Ildong Pharmaceutical Co., Ltd, Seoul, Korea.

\section{Conflict of Interest}

This work was supported by Ildong Pharmaceutical Co., Ltd, Seoul, Korea. No other potential conflict of interest relevant to this article was reported.

\section{Author Contribution}

Conceptualization: Kim JH, Park SJ. Data curation: Kim JH, Choi YJ, Kwon HJ. Formal analysis: Kim JH, Park SJ. Resources: Jung K, Kim SE, Moon W, Park MI. Supervision: Park SJ. Writing - original draft: Kim JH. Writing - review \& editing: Park SJ. Approval of final manuscript: all authors.

\section{ORCID}

Kim JH

https://orcid.org/0000-0002-4272-8003

Choi YJ https://orcid.org/0000-0002-2892-7639

Kwon HJ https://orcid.org/0000-0003-1238-3961

Jung K

Kim SE https://orcid.org/0000-0002-5324-7803

Moon W https://orcid.org/0000-0002-1835-4830

Park MI https://orcid.org/0000-0002-3963-8680 https://orcid.org/0000-0003-2071-6957 
Park SJ

https://orcid.org/0000-0003-3217-5115

\section{Supplementary Material}

Supplementary materials are available at the Intestinal Research website (https://www.irjournal.org).

\section{REFERENCES}

1. US Preventive Services Task Force, Bibbins-Domingo K, Grossman DC, et al. Screening for colorectal cancer: US Preventive Services Task Force recommendation statement. JAMA 2016; 315:2564-2575

2. Inadomi JM, Vijan S, Janz NK, et al. Adherence to colorectal cancer screening: a randomized clinical trial of competing strategies. Arch Intern Med 2012;172:575-582.

3. Denberg TD, Melhado TV, Coombes JM, et al. Predictors of nonadherence to screening colonoscopy. J Gen Intern Med 2005;20:989-995.

4. ASGE Standards of Practice Committee, Fisher DA, Maple JT, et al. Complications of colonoscopy. Gastrointest Endosc 2011; 74:745-752.

5. Ko CW, Riffle S, Shapiro JA, et al. Incidence of minor complications and time lost from normal activities after screening or surveillance colonoscopy. Gastrointest Endosc 2007;65:648656.

6. Cho HS, Han DS, Park HS, et al. The incidence of minor complications and patients' time requirements for colonoscopy. Korean J Gastrointest Endosc 2009;39:205-211.

7. Round JL, Mazmanian SK. The gut microbiota shapes intestinal immune responses during health and disease. Nat Rev Immunol 2009;9:313-323.

8. Nagao-Kitamoto H, Kitamoto S, Kuffa P, Kamada N. Pathogenic role of the gut microbiota in gastrointestinal diseases. Intest Res 2016;14:127-138.

9. Collins SM, Surette M, Bercik P. The interplay between the intestinal microbiota and the brain. Nat Rev Microbiol 2012;10: 735-742.

10. Yu LC, Wei SC, Ni YH. Impact of microbiota in colorectal carcinogenesis: lessons from experimental models. Intest Res 2018;16:346-357.

11. Wong SH, Kwong TNY, Wu CY, Yu J. Clinical applications of gut microbiota in cancer biology. Semin Cancer Biol 2019;55: 28-36.

12. Turnbaugh PJ, Ley RE, Mahowald MA, Magrini V, Mardis ER, Gordon JI. An obesity-associated gut microbiome with increased capacity for energy harvest. Nature 2006;444:1027-
1031.

13. Drago L, Toscano M, De Grandi R, Casini V, Pace F. Persisting changes of intestinal microbiota after bowel lavage and colonoscopy. Eur J Gastroenterol Hepatol 2016;28:532-537.

14. O'Brien CL, Allison GE, Grimpen F, Pavli P. Impact of colonoscopy bowel preparation on intestinal microbiota. PLoS One 2013;8:e62815.

15. Harrell L, Wang Y, Antonopoulos D, et al. Standard colonic lavage alters the natural state of mucosal-associated microbiota in the human colon. PLoS One 2012;7:e32545.

16. Nagata N, Tohya M, Fukuda S, et al. Effects of bowel preparation on the human gut microbiome and metabolome. Sci Rep 2019;9:4042.

17. Jalanka J, Salonen A, Salojärvi J, et al. Effects of bowel cleansing on the intestinal microbiota. Gut 2015;64:1562-1568.

18. Schloss PD, Westcott SL, Ryabin T, et al. Introducing mothur: open-source, platform-independent, community-supported software for describing and comparing microbial communities. Appl Environ Microbiol 2009;75:7537-7541.

19. Quast C, Pruesse E, Yilmaz P, et al. The SILVA ribosomal RNA gene database project: improved data processing and webbased tools. Nucleic Acids Res 2013;41:D590-D596.

20. Edgar RC, Haas BJ, Clemente JC, Quince C, Knight R. UCHIME improves sensitivity and speed of chimera detection. Bioinformatics 2011;27:2194-2200.

21. Cole JR, Wang Q, Cardenas E, et al. The Ribosomal Database Project: improved alignments and new tools for rRNA analysis. Nucleic Acids Res 2009;37:D141-D145.

22. Johansson ME, Gustafsson JK, Holmén-Larsson J, et al. Bacteria penetrate the normally impenetrable inner colon mucus layer in both murine colitis models and patients with ulcerative colitis. Version 2. Gut 2014;63:281-291.

23. Bini EJ, Firoozi B, Choung RJ, Ali EM, Osman M, Weinshel EH. Systematic evaluation of complications related to endoscopy in a training setting: a prospective 30-day outcomes study. Gastrointest Endosc 2003;57:8-16.

24. Ley RE, Bäckhed F, Turnbaugh P, Lozupone CA, Knight RD, Gordon JI. Obesity alters gut microbial ecology. Version 2. Proc Natl Acad Sci U S A 2005;102:11070-11075.

25. Ley RE, Turnbaugh PJ, Klein S, Gordon JI. Microbial ecology: human gut microbes associated with obesity. Nature 2006; 444:1022-1023.

26. Million M, Lagier JC, Yahav D, Paul M. Gut bacterial microbiota and obesity. Clin Microbiol Infect 2013;19:305-313.

27. Jeffery IB, O'Toole PW, Öhman L, et al. An irritable bowel syndrome subtype defined by species-specific alterations in fae- 
cal microbiota. Gut 2012;61:997-1006.

28. Tap J, Derrien M, Törnblom H, et al. Identification of an intestinal microbiota signature associated with severity of irritable bowel syndrome. Gastroenterology 2017;152:111-123.
29. Mariat D, Firmesse O, Levenez F, et al. The Firmicutes/Bacteroidetes ratio of the human microbiota changes with age. BMC Microbiol 2009;9:123. 
See "Effect of gut microbiome on minor complications after a colonoscopy" on page 341-348.

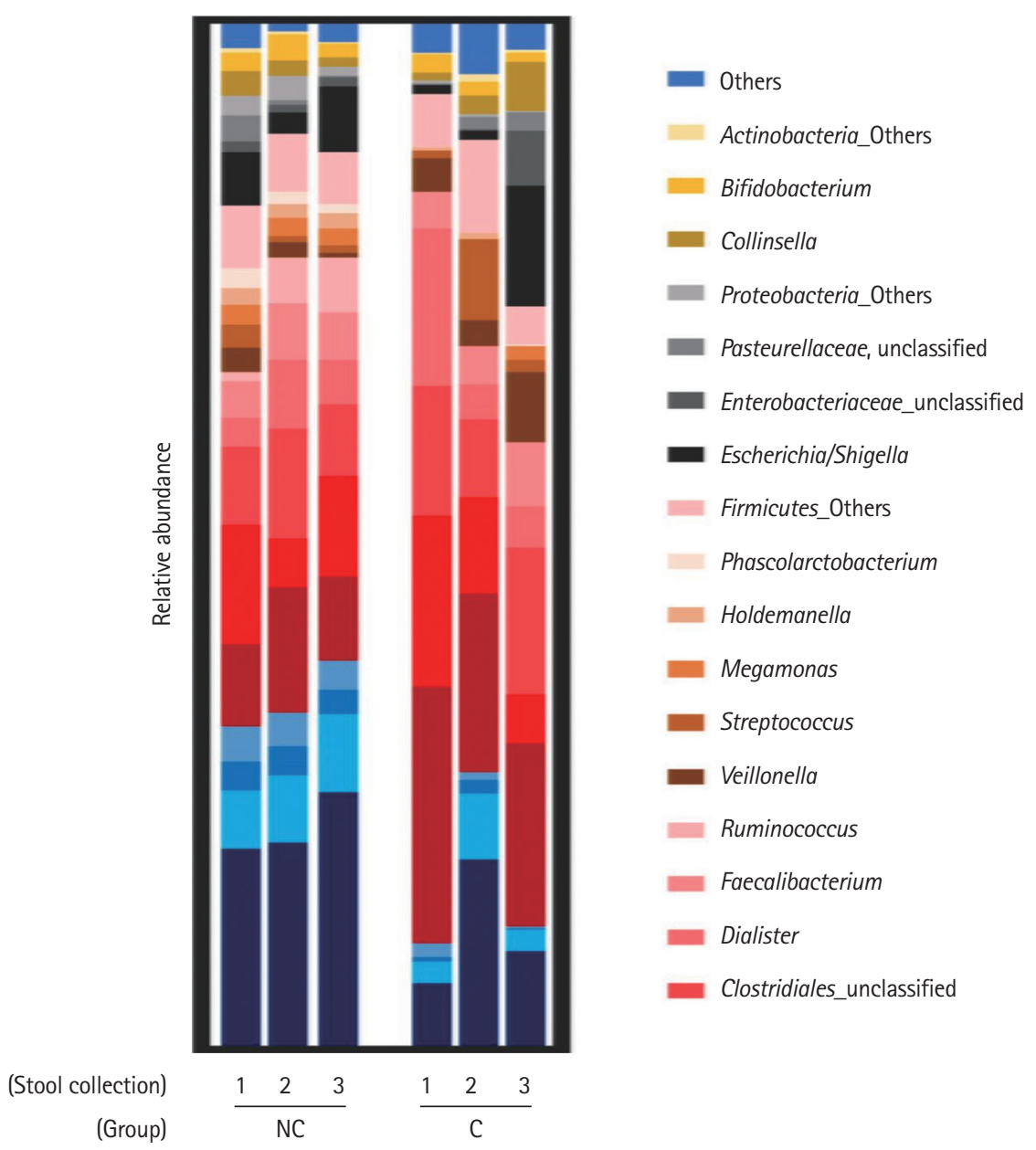

Supplementary Fig. 1. Composition of bacterial communities at the genus level in the 2 groups-no complication group (NC) and complication group (C)-according to the timing of the stool collection (1, before bowel preparation; 2 , on the 7th day after colonoscopy; 3 , on the 28th day after colonoscopy). 
A

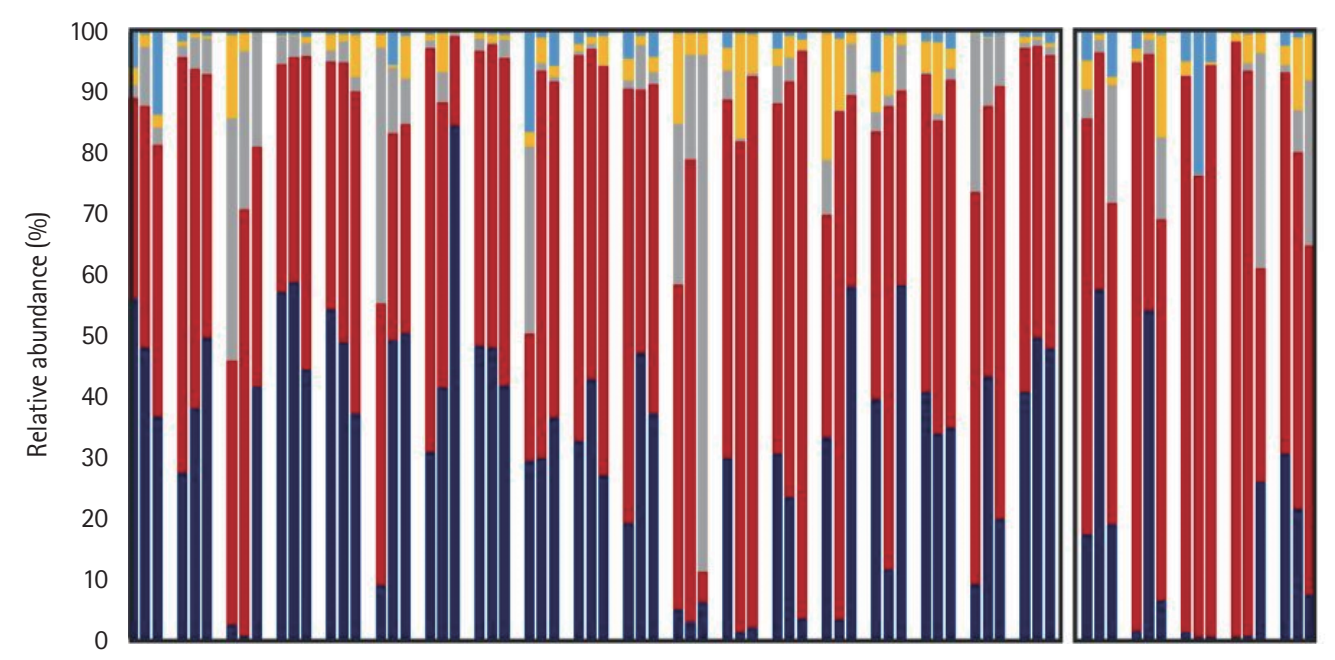

(Stool collection) $\quad 123 \quad 123123123123123123 \quad 123 \quad 123123123123123123123123123123123 \quad 123123123123123$

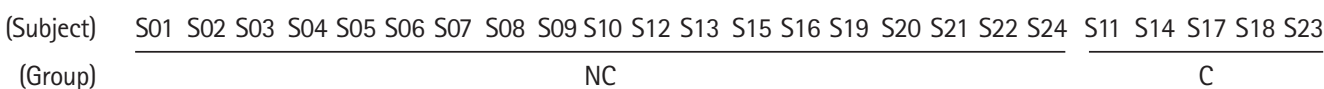

B

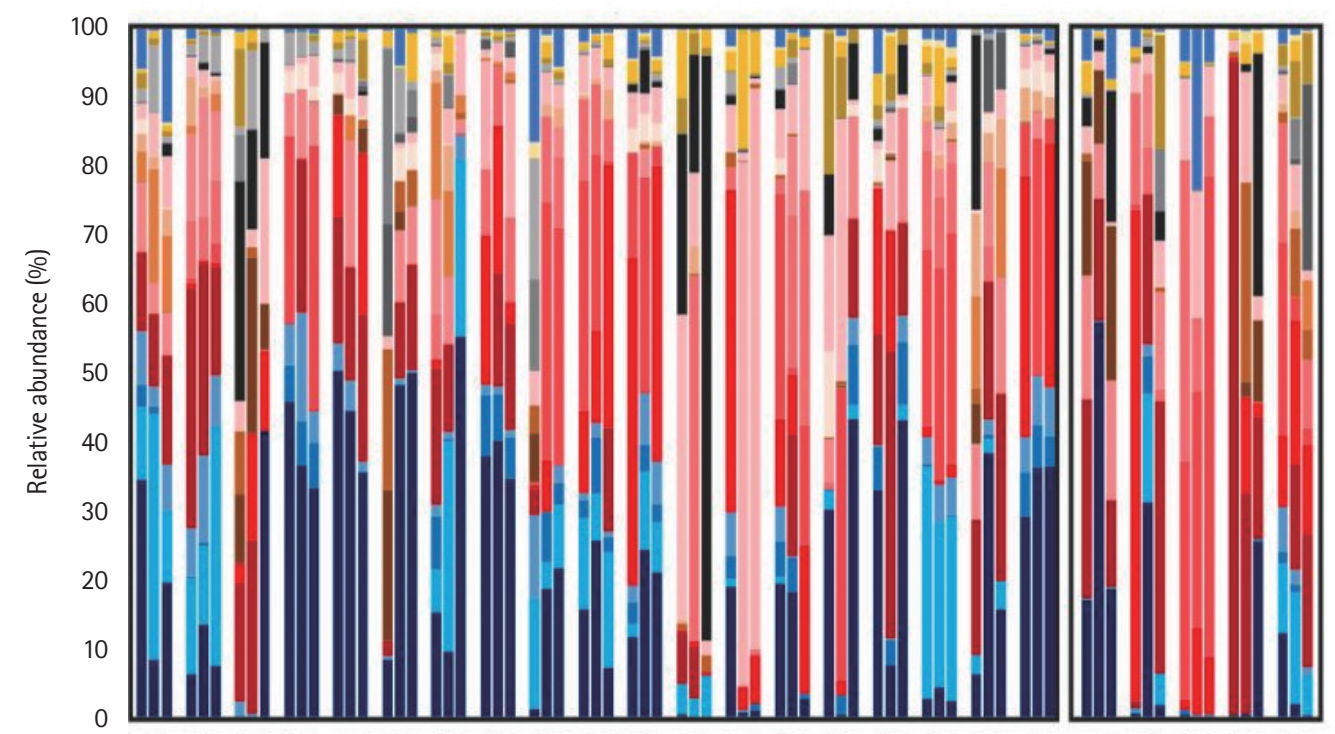

(Stool collection) $\quad 123123123123123123123123123123123123123123123123123123123123123123123123$

$\begin{gathered}\text { (Subject) } \\ \text { (Group) }\end{gathered}$
$\frac{\text { S01 S02 S03 S04 S05 S06 S07 S08 S09 S10 S12 S13 S15 S16 S19 S20 S21 S22 S24 S11 S14 S17 S18 S23 }}{\text { NC }}$

= Others

- Actinobacteria

- Proteobacteria

- Firmicutes

- Bacteroidetes
- Others

* Actinobacteria_0thers

= Bifidobacterium

- Collinsella

= Proteobacteria_Others

- Pasteurellaceae, unclassified

- Enterobacteriaceae_unclassified

- Escherichia/Shigella

= Firmicutes_Others

- Phascolarctobacterium

= Holdemanella

- Megamonas

- Streptococcus

- Veillonella

= Ruminococcus

- Faecalibacterium

- Dialister

- Clostridiales_unclassified

Supplementary Fig. 2. (A) Comparison of the microbial composition at the phylum level in all subjects. (B) Comparison of the microbial

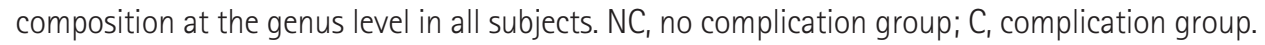

